\title{
Analyzing the Impact of Supply Noise on Jitter in GBPS Serial Links on a Merged I/O-Core Power Delivery Network
}

\author{
Fern-Nee Tan $^{1, \dagger}$ and Sheng Chyan Lee ${ }^{2}$ \\ ${ }^{1}$ Malaysia Design Center, Intel Microelectronics, Penang, 11900, Malaysia \\ ${ }^{2}$ Department of Electronic and Green Technology, Tunku Abdul Rahman University, Kampar, 31900 Malaysia
}

(Received September 13, 2013: Corrected September 30, 2013: Accepted December 2, 2013)

\begin{abstract}
In this paper, the impact of integrating large number of I/O (Input-Output) and Core power Delivery Network (PDN) on a 6 layers Flip-Chip Ball Grid Array (FCBGA) package is investigated. The impact of core induced supply noise on high-speed I/O interfaces, and high-speed I/O interface's supply noise coupling to adjacent high-speed I/O interfaces' jitter impact are studied. Concurrent stress validation software is used to induce SSO noise on each individual I/O interfaces; and at the same time; periodic noise is introduced from Core PDN into the I/O PDN domain. In order to have the maximum coupling impact, a prototype package is designed to merge the $\mathrm{I} / \mathrm{O}$ and Core PDN as one while impact on jitter on each I/O interfaces are investigated. In order to understand the impact of the Core to I/O and I/O to I/O noise, the on-die noise measurements were measured and results were compared with the original PDN where each I/O and Core PDN are standalone and isolated are used as a benchmark.
\end{abstract}

Keywords: Simultaneous switching output (SSO), PCI-Express (PCIe), Serial-ATA (SATA), Universal Serial Bus (USB), Power delivery network (PDN), Flip-Chip Ball grid array (FCBGA)

\section{Introduction}

Most SSO supply noise validation of high speed interfaces such as PCIe, SATA, USB, Core logic etc. is approached as case to case basis. ${ }^{1)}$ Some concurrent test makes use of the device's DFT modes to execute simultaneous tests on different IP blocks like combining scan concurrently with analog tests like RF tuners or serial ATA on an Automated Test Equipment (ATE) is discussed in. ${ }^{2)}$ Layout-aware worst case test pattern ${ }^{3)}$, heuristic method in generating worst case power drop test pattern by accumulating high and lowfrequency effects ${ }^{4}$, and mixed signal validation approach that comprehends both the logic and analog aspects of the circuits during power-up sequence ${ }^{5)}$ were attempts to develop a known worst case test environment to maximize supply noise creation in the validation setup. Recent publication $^{6}$ has questioned the use of scan test mode to create a worst case test pattern "Scan test mode is vulnerable to power supply noise because switching activity is typically three to four times higher than in normal mode as a result of the DFT strategy. This leads to excessive voltage drop during scan testing. In some cases, voltage drop in scan mode has been so excessive that it has resulted in inadvertent logic value toggling and test result corruption".

It's not always clear when these interfaces are put in a common validation eco-system and stressed concurrently, what will be the functionality and performance limiter. This paper describes the impact of integrating large number of I/ $\mathrm{O}$ (Input-Output) and Core power Delivery Network (PDN) on a 6 layers Flip-Chip Ball Grid Array (FCBGA) package. Power supply noise droop of each High Speed I/O interfaces is maximized by implementing a concurrent test in exercising PCIe, SATA and USB to actively transmit data on all the lanes on the electrical board; and at the same time; exerting power gate/ungate noise onto the chip to serve as a natural aggressor from the core logic into the $\mathrm{I} / \mathrm{O}$ interfaces. To make matter worst, a test package is designed to merge all of these high speed interfaces and core logic power rail as one common power rail on the package so that the injected and coupling noise were maximized. The on-die noise measurements were measured and results were compiled to conclude the findings of this study.

${ }^{\dagger}$ Corresponding author

E-mail: fern.nee.tan@intel.com

(C) 2013, The Korean Microelectronics and Packaging Society

This is an Open-Access article distributed under the terms of the Creative Commons Attribution Non-Commercial License(http://creativecommons.org/ licenses/by-nc/3.0) which permits unrestricted non-commercial use, distribution, and reproduction in any medium, provided the original work is properly cited. 


\section{Test Vehicle Design and Concurrent Test Exeprimental Setup}

\subsection{Package Test Vehicle Design}

In order to increase the chances of failure, the original package, Lucerne (Fig.1) was re-designed to merge the high speed serial link power rails together. All the $1.05 \mathrm{~V}$ power rails were merged as one. The power rails involves in the merger includes USB2, PCIe, SATA2, Core, ME, EP and VE and all the digital and analog PLLs of the high speed serial link. This had allowed the high frequency noise coupling from one to another easily via a common power plane. Two test package vehicles were built: Jasper and Milford Sound (Fig. $2 \& 3$ ). Jasper is the test package whereby a conservative merger was done; where I/O and I/ $\mathrm{O}$ power rails were merged, and core to core as one power rail. Meanwhile, Milford Sound is the 2nd test package with aggressive power rail merger. All the $1.05 \mathrm{~V}$ high speed serial link interfaces were merged as one power rail together with the core logic Vcc power rail. Both the test packages used the healthy silicon and both packages have similar footprint as the POR silicon; whereby they were ready to be mounted on the motherboard.

The two significant differences when the power supply rail was merged, was that the power delivery network (PDN) impedance profile and the total amount of on-die capacitance (Cdie) would change. The PDN impedance profile would be lowered, as a larger amount of vias, a larger size of power planes and pins were bounded as one single power rail. Likewise, the Cdie of a merged power rails would be larger. Using one common power rail connectivity, the Cdie of one buffer is easily accessible by the other buffer family using the lower impedance path of the package power plane connection. For example, when Display Port and PCIe are sharing a common power plane design, there is a possibility that the display port's Cdie is visible to PCIe, and PCIe's Cdie is visible to display port, thus giving more margins to high frequency noise suppression when these buffers are operating. On the contrary, the supply noise from one IO buffer is also made easier to couple from one to another.

\subsection{Concurrent Stress Test Setup}

Concurrent test on $1.05 \mathrm{~V}$ merged plane involved the following tests:

- CMM4 pattern was running on all 5x PCIE ports

- SATApower state was exercised to toggle power state change on all 5 SATA ports except OS port

- USB Test packet was exercised to toggle all 14 USB

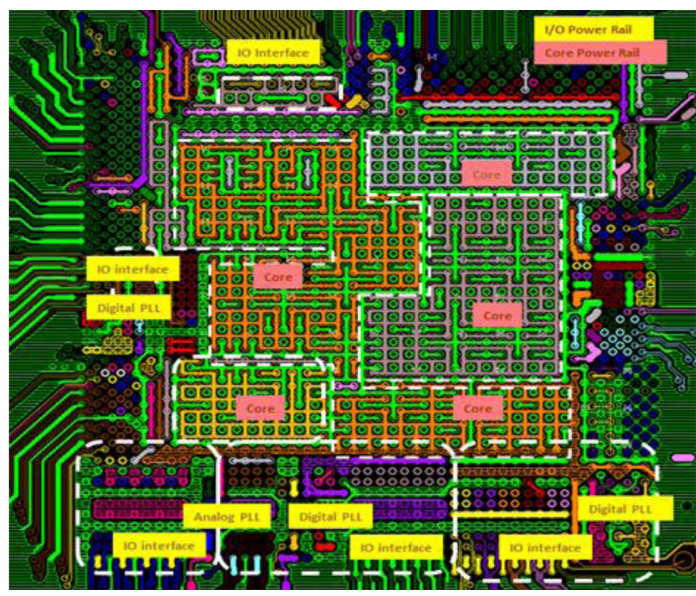

Fig. 1. Lucerne Package: Standalone I/O, PLL and Core PDN.

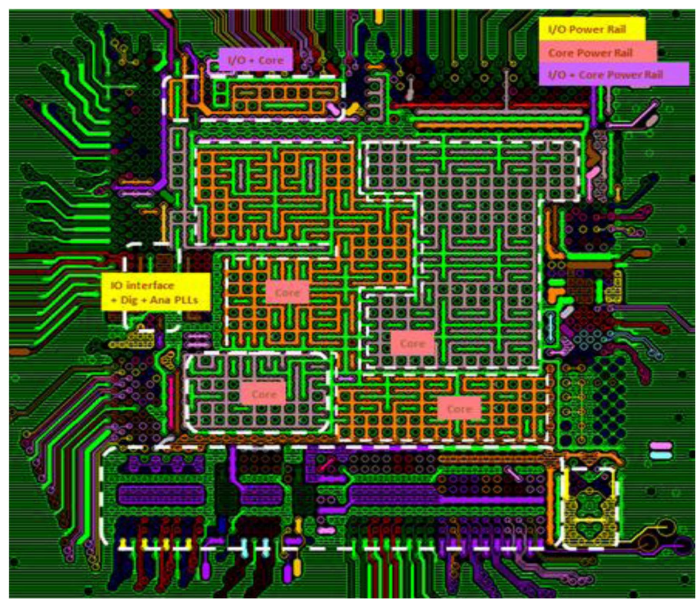

Fig. 2. Jasper Package: I/O and PLL merged \& partial Core merged.

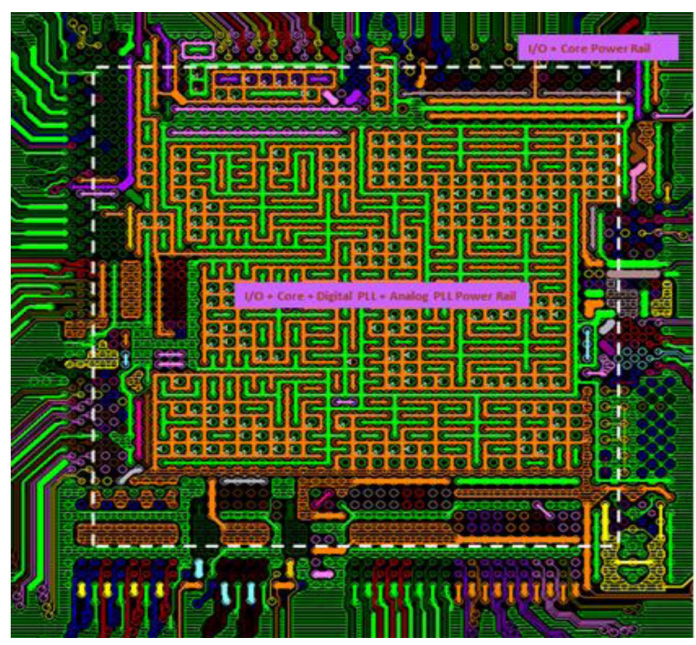

Fig. 3. Milford Sound Package: All Core and I/O are merged.

ports; with eye data collected on port 2

All the tests above are industrial compliance test that must be passed in order to achieve certification of product health. In addition to the industrial compliance tests above, 
Table 1. Industrial product qualification v.s. concurrent stress validation setup.

\begin{tabular}{|c|c|}
\hline \begin{tabular}{|c|}
$\begin{array}{c}\text { Industrial Product Qualification } \\
\text { Validation Setup }\end{array}$ \\
\end{tabular} & $\begin{array}{c}\text { Concurrent Stress Validation } \\
\text { Setup }\end{array}$ \\
\hline One port at a time & $\begin{array}{l}\text { All ports simultaneously (worst port eye- } \\
\text { diagram and jitter is measured) }\end{array}$ \\
\hline One interface at a time & All interfaces simultaneously \\
\hline $\begin{array}{c}\text { Logic and Analog tests are separately } \\
\text { tested }\end{array}$ & $\begin{array}{l}\text { Logic and Analog tests are tested } \\
\text { simultaneously }\end{array}$ \\
\hline $\begin{array}{c}\text { All ports need to pass eye-diagram and } \\
\text { jitter specification }\end{array}$ & $\begin{array}{l}\text { All ports need to pass eye-diagram and } \\
\text { jitter specification }\end{array}$ \\
\hline $\begin{array}{l}\text { One port transmitting at a time } \\
\text { One port receiving at a time }\end{array}$ & $\begin{array}{l}\text { All ports transmitting and receiving } \\
\text { simulataneously }\end{array}$ \\
\hline Analog Front End transmission validation & $\begin{array}{c}\text { Analog Front End transmission and data } \\
\text { bit validity check }\end{array}$ \\
\hline $\begin{array}{c}\text { Analog Front End receiver eye-diagram } \\
\text { validation }\end{array}$ & $\begin{array}{l}\text { Analog Front End received eye-diagram } \\
\text { and digital data bit validity check }\end{array}$ \\
\hline $\begin{array}{c}\text { Power Delivery Noise validation check on } \\
\text { one interface at a time }\end{array}$ & $\begin{array}{c}\text { Power Delivery noise validation check on } \\
\text { all interfaces running at once }\end{array}$ \\
\hline Across all temperature and skew corners & $\begin{array}{c}\text { Cdie measurement on all interfaces on } \\
\text { merged power plane } \\
\text { Selected known worst case temperature } \\
\text { and skew corner only }\end{array}$ \\
\hline
\end{tabular}

additional tests were exercised simultaneously in attempt to inject noise into the system and serve as aggressors to the $\mathrm{I} /$ O circuit. ${ }^{7)}$

To showcase how severe the concurrent test was imposed onto the system for the purpose of this research project, table 1 below compares side by side the difference between industrial product qualification execution and the concurrent stress test setup.

\section{Results and Discussion}

There were 5 packages tested with the concurrent stress test i.e: Lucerne, Jasper, Milford Sound, Jasper and Milford Sound without die-side capacitor (DSC). The packages without DSC were measured to ensure that the existence of DSC would not mask out the power delivery noise and alter the jitter tolerance on the circuit; shifting the circuit's vulnerability to a higher level than the typical range. Table 2 shows the summary of noise and eye data collected for the 3 major interfaces of PCIe, SATA and USB; which all belong to the $1.05 \mathrm{~V}$ high speed serial data interfaces. These $\mathrm{I} / \mathrm{O}$ interfaces were transacting at GBps speed and at the same time stressed with concurrent traffic and power gate/ ungate noise from the digital core logic. From its standalone power plane (Lucerne) to a semi-merged power plane (Jasper) and the fully merged power plane (Milford Sound), PCIE and SATA looked healthy even as the magnitude of noise had increased from $\sim 50 \mathrm{mV}$ to $\sim 100 \mathrm{mV}$; and with onpackage capacitor removed (no DSC). The passing of compliance eye test has indicated that PCIe and SATA had ample margin preventing the eye spec violation or the PDN design did not coincide with the operating clock frequency of the current excitation and thus, the noise on the PDN barely made any significant impact to the circuit operations and degraded its eye performance.

On the other hand, the USB interface had shown intermittent failure although the noise magnitude did not increase much from $67 \mathrm{mV}$ to $80 \mathrm{mV}$. Jasper which had the semi-merged power plane failed all eye spec miserably; although Milford Sound (with DSC) which had the most pessimistic merged power plane was able to survive some of the eye tests; and only failed when the DSC was removed and core noise was introduced. This showed that the failure was intermittent and was not consistent with the noise magnitude increase $(65 \mathrm{mV}$ and $100 \mathrm{mV}$ fail, while $75 \mathrm{mV}$ passes). In other words, the noise magnitude was not the dominant factor that drives the failure of the eye; it was some other parameter that causes the eye failure.

Table 2. PCIe, SATA and USB Noise and eye summary table on 5 test packages.

\begin{tabular}{|c|c|c|c|c|c|c|c|c|c|c|}
\hline \multirow{3}{*}{ Si } & & & & & \multicolumn{6}{|c|}{ Stress Combination } \\
\hline & \multicolumn{2}{|l|}{ A } & \multicolumn{2}{|c|}{$A+B$} & \multicolumn{2}{|c|}{$A+B+C$} & \multicolumn{2}{|c|}{$A+B+C+D$} & \multicolumn{2}{|c|}{$A+B+C+D+E$} \\
\hline & Noise (mV) & Eye & Noise $(\mathrm{mV})$ & Eye & Noise $(\mathrm{mV})$ & Eye & Noise (mV) & Eye & Noise $(\mathrm{mV})$ & Eye \\
\hline \multicolumn{11}{|c|}{ PCI-Express } \\
\hline Lucerne & 52.26 & passed & & & & & & & & \\
\hline Jasper & 57.44 & passed & 64 & passed & 64 & passed & 64.02 & passed & 72 & passed \\
\hline Jasper (no DSC) & 85.16 & passed & 86.25 & passed & 88.19 & passed & 88.28 & passed & 85.58 & passed \\
\hline Milford Sound (with DSC) & 56.83 & passed & 64 & passed & 64 & passed & 64 & passed & 85 & passed \\
\hline Milford Sound (no DSC) & 60.27 & passed & 64 & passed & 69.72 & passed & 70.39 & passed & 101.4 & passed \\
\hline \multicolumn{11}{|c|}{ Serial- ATA } \\
\hline Lucerne & 44.93 & passed & & & & & & & & \\
\hline Jasper & 48 & passed & 48 & passed & 48.38 & passed & 48.58 & passed & 48 & passed \\
\hline Jasper (no DSC) & 51.89 & passed & 51.98 & passed & 51.3 & passed & 53.8 & passed & 54.05 & passed \\
\hline Milford Sound (with DSC) & 52 & passed & 59 & passed & 56 & passed & 60 & passed & 87.84 & passed \\
\hline Milford Sound (no DSC) & 60 & passed & 60.25 & passed & 60.31 & passed & 66.67 & passed & 94.08 & passed \\
\hline \multicolumn{11}{|c|}{ Universal Serial Bus 2.0 (port12) } \\
\hline Lucerne & 67.39 & passed & & & & & & & & \\
\hline Jasper & 68.41 & passed & 60 & passed & 65.34 & passed & 76 & passed & 72.84 & passed \\
\hline Jasper (no DSC) & 76 & failed & 73.08 & failed & 77.33 & failed & 78.59 & failed & 80.69 & failed \\
\hline Milford Sound (with DSC) & 60.17 & m. passed & 60.55 & m. passed & 60.56 & passed & 68.89 & m. passed & 97 & passed \\
\hline Milford Sound (no DSC) & 70.23 & passed & 65 & failed & 72.03 & m. passed & 77.58 & failed & 101.6 & failed \\
\hline
\end{tabular}


Table 3. Eye results on all the USB ports on the system, on different test packages

\section{Legend:}

$A=$ Window $+5 x$ PCIe, $B=A+6 x S A T A$,

$C=B+14 x U S B, D=C+10 x S R A M s, E=C+P M C$.

\begin{tabular}{|c|c|c|c|c|c|c|c|c|c|c|c|c|c|c|c|}
\hline \multicolumn{7}{|c|}{ Electrical Validation USB data (I/Os Concurrent + SRAM + PMC aggressor) } & \multicolumn{2}{|r|}{ Passed } & \multirow{2}{*}{$\begin{array}{l}\text { Waived } \\
\text { Port } 7 \\
\end{array}$} & \multirow{2}{*}{$\begin{array}{l}\text { Failed } \\
\text { Port } 8\end{array}$} & \multirow[b]{2}{*}{ Port 9} & \multirow[b]{2}{*}{ Port 10} & \multirow[b]{2}{*}{ Port 11} & \multirow[b]{2}{*}{ Port 12} & \multirow[b]{2}{*}{ Port 13} \\
\hline & & Port0 & Port1 & Port2 & Port 3 & Port 4 & Port5 & Port 6 & & & & & & & \\
\hline$\overline{\text { Nomina }}$ & Lucerne & Passed & Passed & Passed & Passed & Passed & Passed & Passed & Passed & Passed & Passed & Passed & Passed & Waived & Passed \\
\hline \multirow[t]{3}{*}{$19 "$ cable } & Jasper & Passed & Passed & Passed & Waived & Passed & Passed & Passed & Passed & Passed & Passed & Passed & Passed & Failed & Failed \\
\hline & Milford Sound (SRAM) & Passed & Failed & Failed & Failed & Passed & Passed & Waived & Passed & Waived & Waived & Passed & Passed & Failed & Waived \\
\hline & Milford Sound (PMC) & Passed & Failed & Failed & Failed & Passed & Passed & Waived & Passed & Waived & Passed & Passed & Passed & Failed & Waived \\
\hline
\end{tabular}

\subsection{USB on-die noise to eye impact}

As indicated in the table 2, the test data on USB was based upon the worst case port which was port12 on the system. Further investigation was needed to extend the study to the rest of the ports' eye to see how far the failure was stretched across all ports.

Eye results in table 3 shows that port1-3 were prompted to failure, while port $0,4,5,6 \& 7$ were passing on Milford Sound when core noise were injected. Likewise, port12 was prompted to failure on all Lucerne, Jasper and Milford Sound; whether or not core noise was injected. Not all ports fail in conjunction to core noise injection; and some ports showed more vulnerability than others. Since the ports were all routed on a merged power rail; it was impossible to isolate the power delivery noise on each single port of port $1 / 2 / 3$ from the other; thus, the issue dissection had to be examined from a different perspective other than power delivery noise measurement on each individual port.

To understand the issue better, port 2 (9" cable and 19" cable) and port 12 were selected for a detail eye plot and how it vary with known healthy eye plot.

In figure 4, RMS consecutive jitter was chosen for eye performance analysis. Apple to apple comparison between all passing eye on Port2 with 9" cable against Port2 with 19 " cable and Port 12 with 1" cable had easily shown the difference between the trend of RMS jitter range on passing and failing examples. Red marker indicates eye failure, yellow marker indicates marginally passed eye and no marker means passing eye. Notice that the left graph which showcase all passing eye data had RMS Consecutive Jitter all falling within 20-50ps range, while the case with Port2 and 19" cable had its RMS consecutive jitter skew towards 30-60ps range, Meanwhile, the case with Port 12 and 1" cable had shown a clear indication that any RMS consecutive jitter that exceeded 50ps or fell below 25ps was definitely a failing eye. This is a good graph to indicate that the USB routing (14" of trace length) had already achieved its maximum, and the margin to failure can be marked clearly on this graph. On the case of port2 with 19" cable, the 2 failing eye were encompassed within many marginally passing eye which were also residing within the 30-60ps jitter range. It was difficult to conclude the failing margin using this graph. Either these 2 failing eye data were outliers or the marginally passed cases were somewhat lucky escapes. Comparing port 2 and port12, both RMS consecutive jitter trend was different, whereby port2 19"

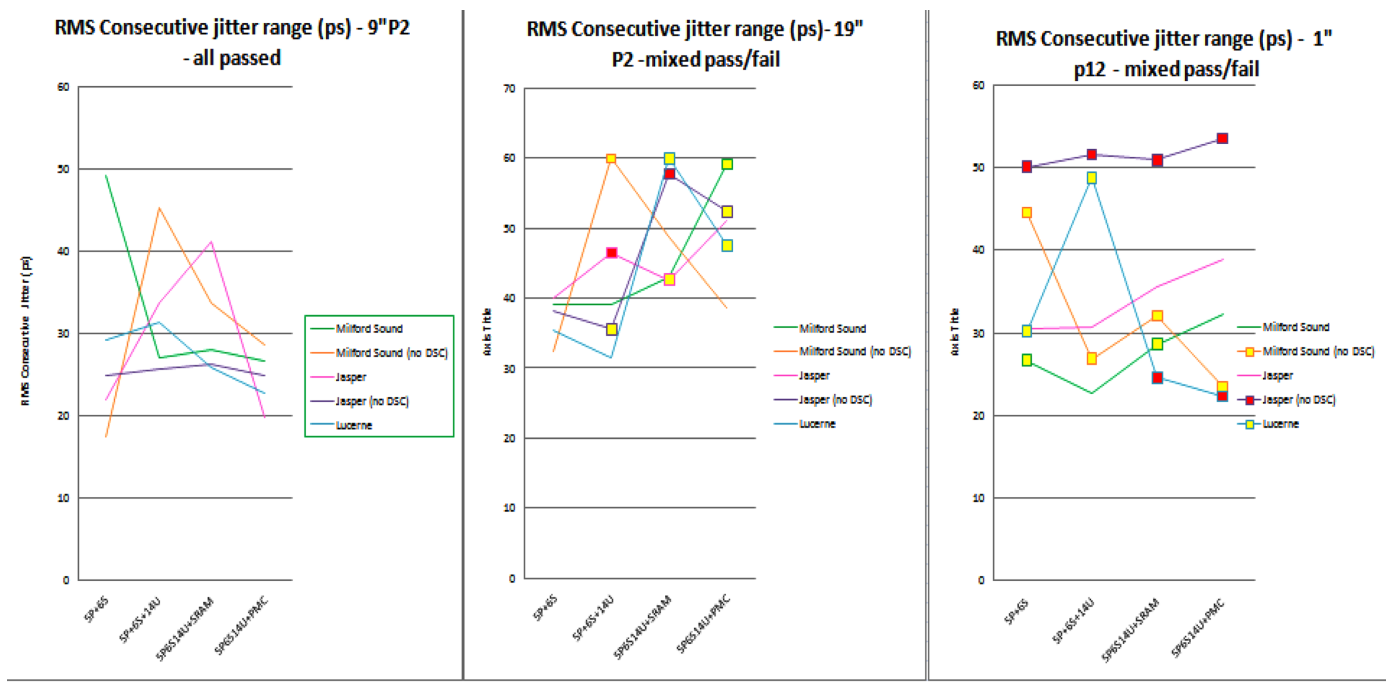

Fig. 4. (Left) RMS Consecutive Jitter on port 2 with 9" cable length to serve as a healthy baseline for comparison for Port 2 -19" cable (middle) and Port 12- 1" cable (right). 

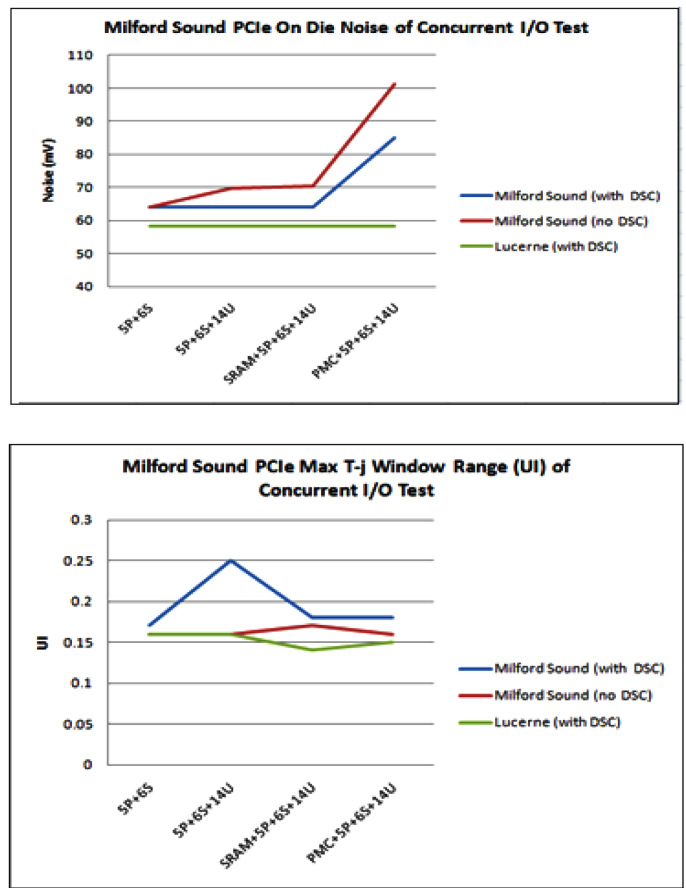

Fig. 5. (Top) PCIe On die-noise (Bottom) PCIe max total-jitter of Milford Sound.

cable obviously can withstand a higher RMS jitter before failing eye than port12 1 " cable. This probably could be explained by the cable having better shielding/grounding compare to traces routed on board.

When comparing these RMS jitter data with on-die noise; Jasper (partially merged power plane) Port12 would not allow on-die noise to go beyond $75 \mathrm{mV}$ before the eye breaks. Meanwhile, Milford Sound (with and without DSC) on port12 1" cable was a lucky escape whereby the eye did not break even-though the on-die noise had stretched beyond $75 \mathrm{mV}$ and reaching almost $100 \mathrm{mV}$; and the RMS jitter was much smaller. Therefore, there is no hard and fast rule to dictate the right spec for peak to peak noise $(\mathrm{mV})$ needed to maintain system health, and on-die noise is not the dominant factor that drives eye failure. To support the argument that on-die noise is not the dominant factor that drives eye failure, more case study is shown below on PCIe

\subsection{Root Causing Eye Failure using System Margining} Approach

By focusing on the last column on table 2, the USB2 port 12 eye diagram had failed consistently when PMC noise was added to the merged power plane; on both Jasper and Milford Sound. For simplicity, Milford Sound was used to reverse margining till the eye on USB2 became passing again.

From experimental perspective, system margining was done through modification of the PMC script to reduce the

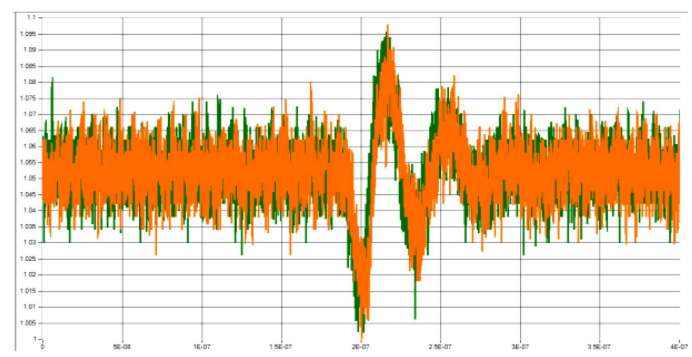

Fig. 6. (Green) Old PMC induced noise (Orange) New PMC induced noise on Milford Sound's PDN.

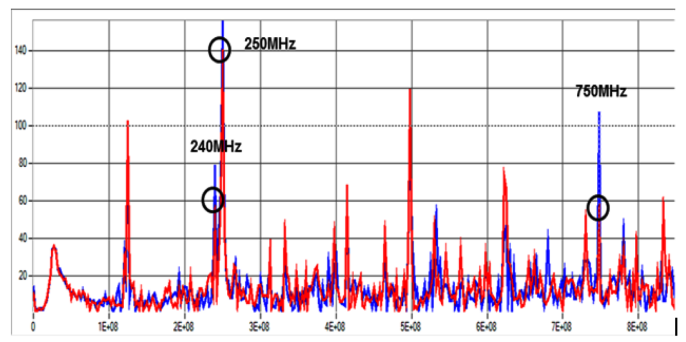

Fig. 7. (Blue) Old PMC induced noise spectrum (Red) New PMC induced noise spectrum on Milford Sound's PDN.

intensity of power gate/ungate activity. Each time, USB eye was measured and checked if it could pass on Jasper and Milford Sound. After the moderation of PMC script to 300000 times slower than original version, all $\mathrm{I} / \mathrm{Os}$ on the system had managed to run successfully passing all eye for at least $30 \mathrm{~min}$ before system hangs. The noise data was compared and shown in figure 6 and 7.

The modification of PMC script had lowered the $240 \mathrm{MHz}$ frequency spectrum and the various other spectrums' intensity and this reduces all the failing eyes to passing.

\section{Summary \& Conclusions}

Minimizing the jitter due to power supply noise is the most important design goal for the power delivery system of High Speed Serial Link interfaces. This paper presented the results of using post-silicon concurrent stress suites to induce noise into the High Speed Serial link interfaces (PCIE, USB and SATA) using two test vehicle packages known as Milford Sound and Jasper. The stress method was by applying the realistic noise generated by concurrent traffic transmitting on each of these interfaces and core power gate/ungate activity; which periodically launch a close to $\sim 100 \mathrm{mV}$ peak to peak noise into these three High Speed Serial Link interfaces. Using $60 \mathrm{mV}$ as our observation baseline, (which was the quoted power delivery target during our design simulation), we observed that USB interface 
suffered the most failure on eye; whereas PCIe and SATA survived with some margin available.

The findings in this investigation were carried out with a new PMC script with noise injected into the High Speed Serial Link's merged power rail on Milford Sound. However, this time, the PMC script had been modified and the $240 \mathrm{MHz}$ spectrum intensity was greatly reduced. Surprisingly, USB started to pass the eye test. Knowing that the $240 \mathrm{MHz}$ was the operating frequency of USB2, it is believed that the eye is greatly influenced by noise spectrum overlaps with its operating frequency. Meanwhile, the noise profile of the new PMC script remains as $\sim 100 \mathrm{mV}$ whereby its magnitude had not reduced.

In conclusion, it is believed that jitter due to power supply noise is real and should be used to set-forth the power delivery target for power delivery network (PDN) analysis. The use of $\mathrm{mV}$ peak to peak noise is inevitably a loose guideline and very often does not define the relationship to the jitter failure well. As shown in the above case study, USB2 eye failed miserably even-though the noise was $65 \mathrm{mV}$ with high $240 \mathrm{MHz}$ component; and if the $240 \mathrm{MHz}$ spectrum is reduced; USB2 eye passed happily even-though the noise had increased to $\sim 100 \mathrm{mV}$. Clearly; $\mathrm{mV}$ noise is no longer a good judging criteria that should be used for power delivery analysis; especially the High Speed Serial Link PDN analysis. Although many will debate about its repeatability on a different product, different platforms; different process corner, it is crucial that the power delivery noise spec be re-defined to encompass noise jitter sensitivity as its performance target.

\section{References}

1. M. G. Gericota, G. R. Alves, M. L. Silva, J. M.Ferreira, "A novel methodology for the concurrent test of partial and dynamically reconfigurable SRAM-based FPGAs", IEEE Proceedings of Design, Automation and Test, EuropeConference and Exhibition 2002; 1126, Mar (2002).

2. S. Molavi, T. McPheeters; "Concurrent Test Implementations", 16th Asian Test Symposium, 2007, 214, Oct (2007).

3. J. Ma, J. Lee, M. Tehranipoor; "Layout-Aware Pattern Generation for Maximizing Supply Noise Effects on Critical Paths", 27th IEEE VLSI Test Symposium, VTS '09, 221, May (2009).

4. I. Polian, A. Czutro, S. Kundu, B. Becker; "Power Droop Testing", International Conference on Computer Design 2006, ICCD 2006, 243, Oct (2007).

5. Pan, Y. C., Mughal, U. A., Rifani, M. C., Wilson, T. M., "Mixed Signal validation of the Intel(R) Pentium (R) 4 microprocessor power-up sequence", Electrical Performance of Electronic Packaging, 2003, 163, Oct (2003).

6. K. Arabi, "Power noise and its impact on production test and validation of SOC devices", 28th VLSI Test Symposium (VTS), 285, April (2010).

7. F. N. Tan, S. C. Lee, F. Abdul Rahman, "Finding the worst case supply noiseexcitation methodology for high speed I/O interfaces", 3rd Asia Symposium on Quality Electronic Design, 161, July (2011). 